\title{
Undergraduate antibiotic stewardship training: Are we leaving our future prescribers 'flapping in the wind'?
}

A multisite survey of South African (SA) medical students' perceptions and knowledge of antibiotic resistance (ABR) and appropriate prescribing by Wasserman et al., ${ }^{[1]}$ published in this edition of the $S A M J$, demonstrates that our final-year medical undergraduates are clearly inadequately prepared for practice. Moreover, crucial gaps in knowledge and poor understanding of antibiotic stewardship (AS) and infection control, vary geographically. This first-in-kind crosssectional survey of the preparedness of final-year medical students to prescribe antibiotics identified several enablers that necessitate alternative educational strategies and interventions that could decisively affect the prescribing by graduates. In keeping with a recent study of final-year SA pharmacy students, ${ }^{[2]}$ by far the majority of respondents reported that they would prefer more education on appropriate antibiotic use. In fact, less than two-thirds reported being familiar with the term 'antibiotic stewardship'. More disconcerting, only a third of respondents felt confident to prescribe antibiotics, with similar proportions across institutions.

Prescribing confidence was significantly associated with several determinants, such as access to antibiotic prescribing guidelines, familiarity with AS, and more frequent contact with infectious diseases specialists. In contrast, the least perceived measures to improve preparedness were more contact with microbiologists, didactic lectures and the use of computer-based tutorials. Perhaps this suggests that however well-meaning, stewardship-related diagnostic or therapeutic teaching in lectures does not translate into clinical practice. No significant associations were found between knowledge scores and sources of educational information, except paradoxically, lower scores among those students who reported reading medical journals.

While the majority of respondents agreed that antibiotics are overused and ABR is a significant problem in SA, for both of these determinants it was perceived to be less of a problem in their own institutions. However, the view of 'not in my backyard, ${ }^{[3]}$ which suggests that others overprescribe antibiotics and drive resistance, may contribute to inappropriate prescribing behaviours. ${ }^{[4,5]}$ The underlying cognitive bias for the students' contradictory views need to be explored and may represent yet another enabler to affect behaviour.

In this regard, we need to capitalise on undergraduate students' perceptions as a matter of priority. While the study did not map AS education among the institutions, ${ }^{[6]}$ existing education in SA medical schools may be augmented by standardisation, focusing on equipping young doctors with the necessary confidence and skills in appropriate antibiotic prescribing at an early stage of their careers, as well as implementing interventions to modify perceptions and behaviours. To mitigate the disparate education that our undergraduates are receiving, consideration should be given to establishing a national collaborative undertaking, similar to the Appropriate Prescribing for Tomorrow's Doctors (APT) project, which was formed to address similar needs at undergraduate level in Scotland and the UK. ${ }^{[7]}$ The formation of a joint SA working group can facilitate the process of employing standardised learning outcomes of a core consensus stewardship curriculum. Education within such a framework may permit flexibility of delivery, while allowing individual institutions to generate the methods by which the outcomes are achieved, without requiring rigid adherence to a set of standard teaching methods. ${ }^{[7]}$

Ultimately, the principles of ABR and appropriate use are universal across a 'one health' platform. It is, therefore, important that other cadres of healthcare professionals should be included. As a result, surveys to identify gaps in undergraduate education (for which a paucity of data exists internationally for veterinarians and dentists) are required to strengthen undergraduate training and ultimately improve prescribing practices of all healthcare providers in SA. The same applies to nurse practitioners, who are an important cadre of healthcare professional with a proven track record in antibiotic prescribing in SA communities, ${ }^{[8]}$ in addition to intensive-care nurses, who already play a pivotal role in multidisciplinary hospital-based stewardship programmes. ${ }^{[9,10]}$ Opportunities to improve baseline knowledge of pharmacists in SA have already been identified, ${ }^{[2,10]}$ and work to assess barriers and enablers to reduce variability in stewardship education at the eight SA pharmacy schools has begun.

Notably, dentists who are not a focus of formal stewardship programmes yet, prescribe a substantial proportion of antibiotics in the outpatient setting. In the UK, dentists prescribe $\sim 10 \%$ of antibiotics dispensed in community pharmacies but, similarly to clinicians, they often do so against clear clinical guidelines. ${ }^{[1]}$ Based on prescription data in Canada, dental prescribing increased by $62.2 \%$ (1996 - 2013), with proportionate contribution to community consumption increasing from $6.7 \%$ to $11.3 \%$ of antibiotic prescriptions over the same period. ${ }^{[12]}$ Although data in terms of an SA perspective are not available yet, given the unique local healthcare challenges, there is no reason to believe that it will be much different. Therefore, targeting undergraduate dental students, including dental therapists, who have restricted prescribing privileges, is an important step in minimising overall antibiotic consumption.

In animal health, the extensive, non-therapeutic use of antibiotics in food animals for growth promotion, metaphylaxis and prophylaxis raise serious concerns. This issue highlights the vital public health role and tremendous responsibility of veterinarians, who are often tasked with assessing and advising with regard to the conflicting objectives of upholding animal welfare and food security on the one hand, and keeping the interests of human health in mind on the other. Moreover, the major increase in the use of antibiotics in companion animals and recent evidence of the reciprocal transfer of resistant pathogens between pets and their owners should prompt urgent attention to AS in this context. ${ }^{[13]}$ Consequently, the need for a structured curriculum regarding ABR to bridge a similar gap for veterinary students and practitioners is therefore also recognised.

Once an outcome-based core curriculum is established, the question remains how and in what format it should be delivered. Based on the students' responses in this survey regarding the usefulness of various educational modalities and sources, it appears that passive education to teach fundamental stewardship principles should be complemented with active educational approaches, such as interactive e-learning. For example, recognising the crucial role of veterinarians in mitigating ABR, the Centers for Disease Control and Prevention (CDC), USA, have funded the development of a suite of educational materials to promote the responsible veterinary use of antibiotics. ${ }^{[14]}$ The format, an open-access, web-based multimedia curriculum regarding $A B R$ in veterinary practice, was designed for integration into existing veterinary medical courses, but was also a resource for practising veterinarians. Such innovative teaching methods could be developed for SA and shared between medical, dental, pharmacy and nursing schools to standardise AS education with reliable and 
effective sources of information, aiming to improve the overall use of antimicrobials and, as a result, attempt to reduce the current burden of ABR.

A key strategy in facilitating appropriate antibiotic prescribing is the early introduction of the relevant knowledge, concepts and skills into undergraduate curricula. Findings from this study of undergraduate medical students promote the need for drastic educational measures in response. The promising enablers identified by Wasserman et al. ${ }^{[1]}$ combined with the considerable level of attention to ABR, may provide momentum in the right direction when it comes to setting our future stewards on a course of prescribing confidence for the rest of their careers.

\section{Adrian Brink}

Ampath National Laboratory Services, Milpark Hospital, Johannesburg; and Division of Infectious Diseases and HIV Medicine, Department of Medicine, Groote Schuur Hospital and Faculty of Health Sciences, University of Cape Town, South Africa

brinka@ampath.co.za

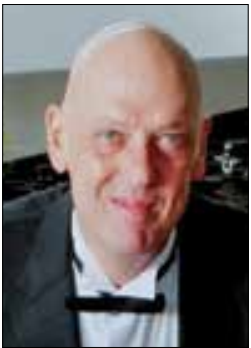

\section{Johan Schoeman}

Department of Companion Animal Clinical Studies, Faculty of Veterinary Science, University of Pretoria, South Africa

\section{George Muntingh}

Department of Pharmacology, School of Medicine, Faculty of Health Sciences, University of Pretoria, South Africa
1. Wasserman S, Potgieter S, Shoul E, et al. South African medical students' perceptions and knowledge about antibiotic resistance and appropriate prescribing: Are we providing adequate training to future prescribers? S Afr Med J 2017;107(5):405-410. https://doi.org/10.7196/SAMJ.2017.v107i5.12370

2. Burger M, Fourie J, Loot D, et al. Knowledge and perceptions of antimicrobial stewardship concepts 2. Burger M, Fourie J, Loot D, et al. Knowledge and perceptions of antimicrobial stewardship concepts
among final year pharmacy students in pharmacy schools across South Africa. S Afr J Infect Dis 2016;1(3):84-90.

3. Not in my backyard (NIMBY). http://dictionary.cambridge.org/dictionary/english/nimby?a=british (accessed 16 March 2016).

4. Abbo L, Sinkowitz-Cochran R, Smith L, et al. Faculty and resident physicians' attitudes, perceptions, and knowledge about antimicrobial use and resistance. Infect Control Hosp Epidemiol 2011;32(7):714-718.

5. McCullough AR, Rathbone J, Parekh S, et al. Not in my backyard: A systematic review of clinicians' knowledge and beliefs about antibiotic resistance. J Antimicrob Chemother 2015;70(9):2465-2473. https://doi.org/10.1093/jac/dkv164

6. Castro-Sánchez H, Drumright LN, Gharbi M, et al. Mapping antimicrobial stewardship in undergraduate medical, dental, pharmacy, nursing and veterinary education in the United Kingdom. PLoS ONE 2016;11(2):e0150056. https://doi.org/10.1371/journal.pone.0150056

7. Paterson Davenport AL, Davey PG, Ker JS; on behalf of the BSAC Undergraduate Education Working Party. An outcome-based approach for teaching prudent antimicrobial prescribing to undergraduate medical students: Report of a Working Party of the British Society for Antimicrobial Chemotherapy. J Antimicrob Chemother 2005;56(1):196-203. https://doi.org/10.1093/jac/dki126

8. Brink AJ, van den Bergh D, Mendelson M, et al. Passing the baton to pharmacists and nurses: New models of antibiotic stewardship for South Africa? S Afr Med J 2016;106(10):947-948. https://doi. org/10.7196/SAMJ.2016.v106i10.11448

9. Olans RN, Olans RD, DeMaria A. The critical role of the staff nurse in antimicrobial stewardship unrecognized, but already there. Clin Infect Dis 2016;62(1):84-89. https://doi.org/10.1093/cid/civ697

10. Schellack N, Pretorius R, Messina AP. 'Esprit de corps': Towards collaborative integration of pharmacists and nurses into antimicrobial stewardship programmes in South Africa. S Afr Med J 2016;106(10):973974. https://doi.org/10.7196/SAMJ.2016.v106i10.11468

11. Elouafkaoui P, Young L, Newlands R, et al. An audit and feedback intervention for reducing antibiotic prescribing in general dental practice: The RAPiD cluster randomised controlled trial. PLoS Med 2016;13(8):e1002115. https://doi.org/10.1371/journal.pmed.1002115

12. Marra F, George D, Chong M, et al. Antibiotic prescribing by dentists has increased. Why? J Am Dental Assoc 2016:147(5):320-327. https://doi.org/10.1016/j.adaj.2015.12.014

13. Wieler LH, Ewers C, Guenther S, et al. Methicillin-resistant staphylococci (MRS) and extendedspectrum $\beta$-lactamases (ESBL)-producing Enterobacteriaceae in companion animals: Nosocomial infections as one reason for the rising prevalence of these potential zoonotic pathogens in clinical samples. Int J Med Microbiol 2011;301(8):635-641. https:// doi.org/10.1016/.ijmm.2011.09.009

14. Gordoncillo MJN, Bender J, Noffsinger J, et al. Developing an open-access antimicrobial resistance learning site for veterinary medical students. J Vet Med Educ 2011;38(4):404-407. https://doi.org/10.3138/ jvme.38.4.40

S Afr Med J 2017;107(5):357-358. DOI:10.7196/SAMJ.2017.v107i5.12496 